SUPPLEMENTARY DATA:

\title{
RESOLUTION OF THE EARLY JURASSIC ACTINOPTERYGIAN FISH PACHYCORMUS AND A DISPERSAL HYPOTHESIS FOR PACHYCORMIFORMES
}

LOVISA WRETMAN, ${ }^{*}, 1$ HENNING BLOM, ${ }^{1}$ and BENJAMIN P. KEAR ${ }^{2}$; ${ }^{1}$ Department of Organismal Biology, Uppsala University, Norbyvägen 18A, 752 36, Uppsala, Sweden, lovisa.wretman@ebc.uu.se; henning.blom@ebc.uu.se; ${ }^{2}$ Museum of Evolution, Uppsala University, Norbyvägen 16, Uppsala SE-752 36, Sweden, benjamin.kear@em.uu.se 


\section{MEASUREMENTS}

TABLE S1. Maximum body length and cranium measurements ( $\mathrm{mm}$ ) for specimens of Pachycormus curtus $(\mathrm{n}=8)$, P. macropterus $(\mathrm{n}=3), P$. bollensis $(\mathrm{n}=6)$, and Pachycormus sp. $(\mathrm{n}=14)$.

\begin{tabular}{|c|c|c|c|c|}
\hline Catalog number & Species & Maximum Body Length & Cranium & Ratio \\
\hline GPIT/74332-4 & P. curtus & 256 & 66 & $3.9: 1$ \\
\hline SMNS 87771 & P. curtus & 200 & 57 & $3.5: 1$ \\
\hline SMNS 96618 & P. curtus & 260 & 55 & $4.7: 1$ \\
\hline SMNS 55300 & P. curtus & 380 & 94 & $4: 1$ \\
\hline SMNS 87762 & P. curtus & 310 & 80 & $3.8: 1$ \\
\hline PMU 24211 & P. curtus & 380 & 94 & $4: 1$ \\
\hline UMH unregistered specimen 1 & P. curtus & 500 & 125 & $4: 1$ \\
\hline UMH unregistered specimen 2 & P. curtus & 370 & 105 & $3.5: 1$ \\
\hline UMH unregistered specimen 3 & P. macropterus & 530 & 135 & $3.9: 1$ \\
\hline P7569 (BMNH) & P. macropterus & 670 & 179 & $3.7: 1$ \\
\hline P12913 (BMNH) & P. macropterus & 520 & 135 & $3.9: 1$ \\
\hline GPIT/OS/777 & P. bollensis & 850 & 250 & $3.4: 1$ \\
\hline GPIT/OS/776 & P. bollensis & 890 & 210 & $4.2: 1$ \\
\hline UMH unregistered specimen 4 & P. bollensis & 890 & 235 & $3.8: 1$ \\
\hline UMH unregistered specimen 5 & P. bollensis & 750 & 174 & $4.3: 1$ \\
\hline UMH unregistered specimen 6 & P. bollensis & 750 & 190 & $3.9: 1$ \\
\hline PMU 24210 & P. bollensis & 860 & 235 & $3.7: 1$ \\
\hline SMNS 87772 & Pachycormus sp. & 420 & 120 & $3.5: 1$ \\
\hline SMNS 87763 & Pachycormus sp. & 300 & 74 & $4.1: 1$ \\
\hline SMNS 95835 & Pachycormus sp. & 410 & 106 & $3.9: 1$ \\
\hline SMNS 65230 & Pachycormus sp. & 420 & 110 & $3.8: 1$ \\
\hline SMNS 51031 & Pachycormus sp. & 575 & 140 & $4.1: 1$ \\
\hline SMNS 18189 & Pachycormus sp. & 245 & 74 & $3.3: 1$ \\
\hline UMH unregistered specimen 7 & Pachycormus sp. & 1020 & 275 & $3.7: 1$ \\
\hline UMH unregistered specimen 8 & Pachycormus sp. & 635 & 168 & $3.8: 1$ \\
\hline UMH unregistered specimen 9 & Pachycormus sp. & 483 & 130 & $3.7: 1$ \\
\hline UMH unregistered specimen 10 & Pachycormus sp. & 490 & 90 & $5.4: 1$ \\
\hline UMH unregistered specimen 11 & Pachycormus sp. & 350 & 88 & $4: 1$ \\
\hline UMH unregistered specimen 12 & Pachycormus sp. & 920 & 190 & $4.8: 1$ \\
\hline UMH unregistered specimen 13 & Pachycormus sp. & 850 & 220 & $3.9: 1$ \\
\hline UMH unregistered specimen 14 & Pachycormus sp. & 895 & 210 & $4.7: 1$ \\
\hline
\end{tabular}


TABLE S2. Cranium and preorbital measurements $(\mathrm{mm})$ for specimens of Pachycormus curtus $(\mathrm{n}=8), P$. macropterus $(\mathrm{n}=5)$ P. bollensis $(\mathrm{n}=7)$, and Pachycormus sp. $(\mathrm{n}=17)$.

\begin{tabular}{|c|c|c|c|c|}
\hline Catalog number & Species & Cranium & Preorbital & Ratio \\
\hline GPIT/74332-4 & P. curtus & 66 & 29 & $2.3: 1$ \\
\hline SMNS 87771 & P. curtus & 57 & 24 & $2.4: 1$ \\
\hline SMNS 96618 & P. curtus & 55 & 15 & $3.7: 1$ \\
\hline SMNS 55300 & P. curtus & 94 & 27 & $3: 1$ \\
\hline SMNS 87762 & P. curtus & 80 & 38 & $2: 1$ \\
\hline PMU 24210 & P. curtus & 94 & 37 & $2.5: 1$ \\
\hline UMH unregistered specimen 1 & P. curtus & 125 & 36 & $3: 1$ \\
\hline UMH unregistered specimen 2 & P. curtus & 105 & 37 & $2.2: 1$ \\
\hline GPIT/773 & P. macropterus & 139 & 47 & $3: 1$ \\
\hline GPIT/774 & P. macropterus & 110 & 43 & $2.5: 1$ \\
\hline UMH unregistered specimen 3 & P. macropterus & 135 & 50 & $3: 1$ \\
\hline P7569 (BMNH) & P. macropterus & 179 & 73 & $2.5: 1$ \\
\hline P12913 (BMNH) & P. macropterus & 135 & 44 & $3: 1$ \\
\hline GPIT/OS/777 & P. bollensis & 250 & 100 & $2.5: 1$ \\
\hline GPIT/OS/776 & P. bollensis & 210 & 80 & $2.5: 1$ \\
\hline UMH unregistered specimen 4 & P. bollensis & 235 & 85 & $3: 1$ \\
\hline UMH unregistered specimen 15 & P. bollensis & 168 & 60 & $3: 1$ \\
\hline UMH unregistered specimen 5 & P. bollensis & 174 & 70 & $2.5: 1$ \\
\hline UMH unregistered specimen 6 & P. bollensis & 190 & 70 & $2.5: 1$ \\
\hline PMU P1191 & P. bollensis & 235 & 91 & $2.5: 1$ \\
\hline SMNS 87769 & Pachycormus sp. & 180 & 76 & $2.5: 1$ \\
\hline SMNS 87772 & Pachycormus sp. & 120 & 40 & $3: 1$ \\
\hline SMNS 87763 & Pachycormus sp. & 74 & 30 & $2.5: 1$ \\
\hline SMNS 87760 & Pachycormus sp. & 50 & 16 & $3: 1$ \\
\hline SMNS 95835 & Pachycormus sp. & 106 & 45 & $2.5: 1$ \\
\hline SMNS 53425 & Pachycormus sp. & 240 & 90 & $2.5: 1$ \\
\hline SMNS 65230 & Pachycormus sp. & 110 & 60 & $2: 1$ \\
\hline SMNS 51031 & Pachycormus sp. & 140 & 54 & $3: 1$ \\
\hline SMNS 18189 & Pachycormus sp. & 74 & 29 & $2.5: 1$ \\
\hline UMH unregistered specimen 7 & Pachycormus sp. & 275 & 90 & $3: 1$ \\
\hline UMH unregistered specimen 8 & Pachycormus sp. & 168 & 54 & $3: 1$ \\
\hline UMH unregistered specimen 9 & Pachycormus sp. & 130 & 60 & $2.2: 1$ \\
\hline UMH unregistered specimen 10 & Pachycormus sp. & 90 & 43 & $2.1: 1$ \\
\hline UMH unregistered specimen 11 & Pachycormus sp. & 88 & 37 & $2.4: 1$ \\
\hline UMH unregistered specimen 12 & Pachycormus sp. & 190 & 80 & $2.4: 1$ \\
\hline UMH unregistered specimen 13 & Pachycormus sp. & 220 & 80 & $2.8: 1$ \\
\hline UMH unregistered specimen 14 & Pachycormus sp. & 210 & 85 & $2.5: 1$ \\
\hline
\end{tabular}

TABLE S3. Opercular measurements $(\mathrm{mm})$ for specimens of Pachycormus curtus $(\mathrm{n}=6)$ and P. macropterus $(\mathrm{n}=3)$.

\begin{tabular}{llcccc}
\hline Catalog number & Species & Length & Height & Ratio & Position of ventral angle \\
\hline P2037a (BMNH) & P. curtus & 40 & 20 & $2: 1$ & Midlength \\
$32435(\mathrm{BMNH})$ & P. curtus & 31.7 & 15.8 & $2: 1$ & Midlength \\
$32439(\mathrm{BMNH})$ & P. curtus & 41.2 & 20.6 & $2: 1$ & Midlength \\
$32427(\mathrm{BMNH})$ & P. curtus & 32.3 & 15.4 & $2.1: 1$ & Midlength \\
$32425(\mathrm{BMNH})$ & P. curtus & 32.3 & 20 & $1.6: 1$ & Midlength \\
P12913 (BMNH) & P. macropterus & 48.8 & 32.4 & $1.5: 1$ & Midlength \\
P7569 $(\mathrm{BMNH})$ & P. macropterus & 70 & 38 & $1.8: 1$ & Midlength \\
GPIT/774 & P. macropterus & 36 & 20 & $1.8: 1$ & Midlength \\
GPIT/OS/776 & P. bollensis & 45 & 25 & $1.8: 1$ & Anterior to midlength \\
\hline
\end{tabular}



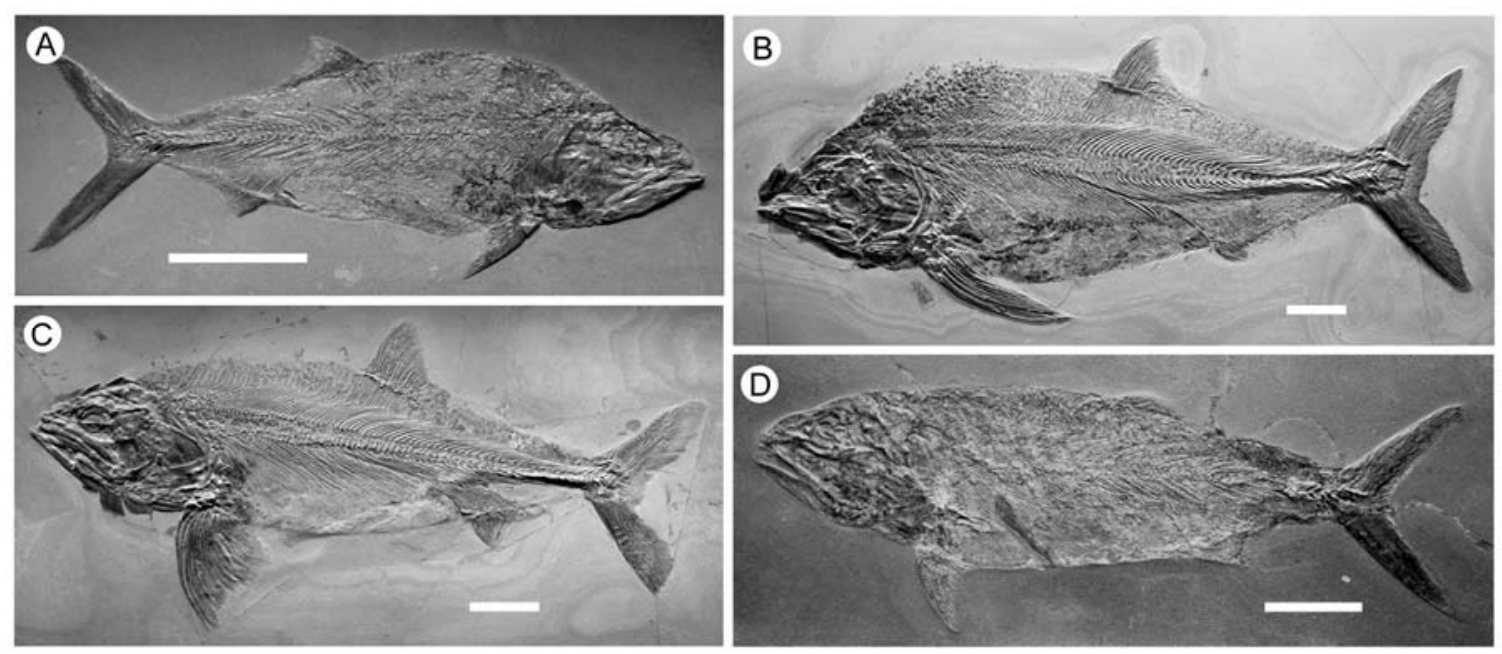

FIGURE S1. Examples of Pachycormus from the UMH illustrating morphotype variation: (A) unregistered specimen 2, P. curtus; (B) unregistered specimen 5, P. bollensis; (C) unregistered specimen 6, P. bollensis; (D) unregistered specimen 11, Pachycormus sp. Scales equal $50 \mathrm{~mm}$. Additional photographs of other UMH specimens are available from the authors upon request.

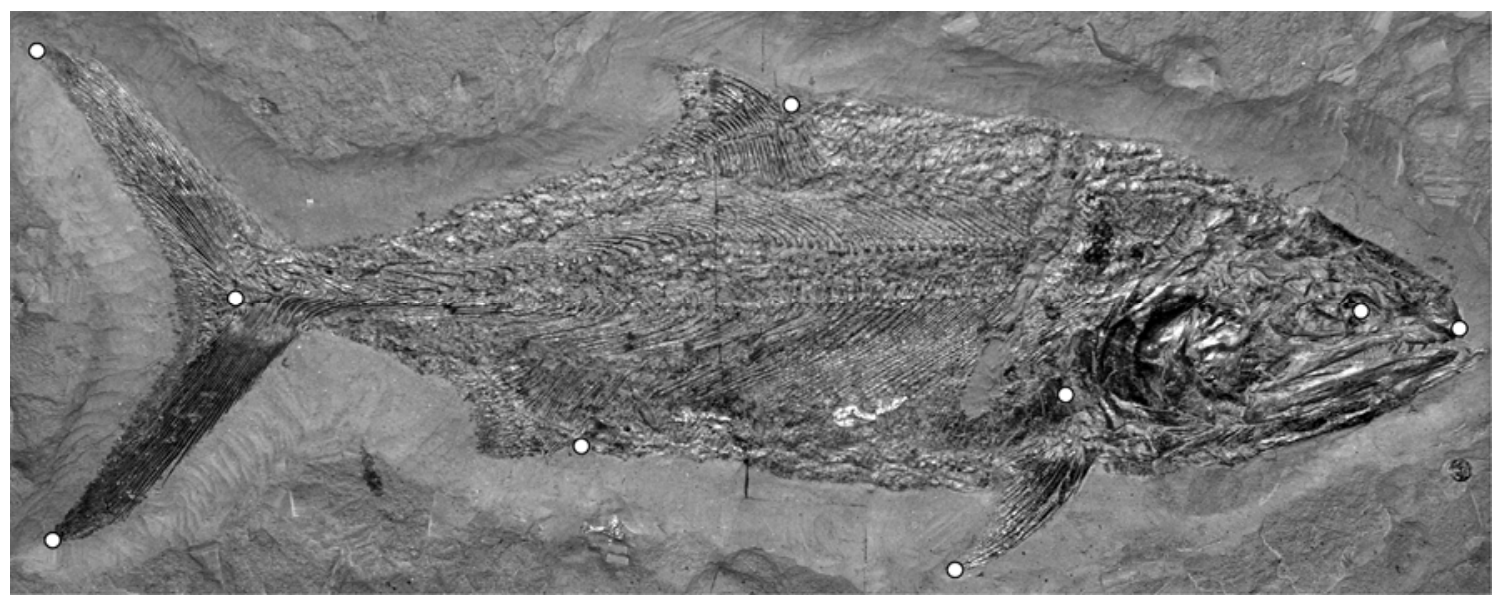

FIGURE S2. Fixed anatomically corresponding landmarks designated for the relative warps analysis. Only skeletally complete specimens $(\mathrm{n}=14)$ were assessed, with $2 / 14(7 \%)$ lacking fully intact pectoral and caudal fins. Representative species labelled as Pachycormus curtus in the FMHS (unregistered specimen displayed behind glass and not accessible for study).

TABLE S4. Morphological variance contributing to relative warps.

\begin{tabular}{lcc}
\hline RW & \% variance & Cummulative $\%$ \\
\hline 1 & $36.09 \%$ & $36.09 \%$ \\
2 & $27.62 \%$ & $63.71 \%$ \\
3 & $15.14 \%$ & $78.85 \%$ \\
4 & $6.49 \%$ & $85.34 \%$ \\
5 & $5.47 \%$ & $90.80 \%$ \\
\hline
\end{tabular}




\section{PHYLOGENENTIC AND PALEOBIOGEOGRAPHICAL DATA SCORES}

Revised character scores (NEXUS format) for the Friedman (2012) pachycormiform matrix.

Pachycormus_macropterus

00101100000-00-0111000?0001010100001001-0101-

01100101100000000100001?01010000000100000000001110100?111001001101110?11-000

Pachycormus_curtus

00101100000-00-0111000?0001010100001001-0101-

01100101100000000100001?01010000000100000000001110(01)00?111001001101110?11-000

Pachycormus_bollensis

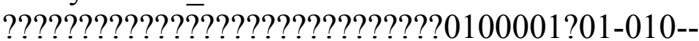

01100?011000001001000011??0???0????100??????0???10100?11100??01???110011-000

TABLE S5. Paleobiogeographical area state codes for pachycormiform taxa. A: Boreal Tethys; B: Western Interior Seaway; C: Eromanga Seaway; D: Western Pacific rim; E: Hispanic corridor; F: Proto-Atlantic; G: Equatorial Tethys; H: Eastern Pacific rim; I: Australasian Tethys; J: Eastern Atlantic rim. Reference literature: Mainwaring (1978); Stewart (1988); McNamara et al. (1993); Lambers (1992); Kear (2007); Kear et al. (2009); Friedman et al. (2010); Liston (2010); Arratia and Scultze (2013); Friedman et al. (2013); Bazzi et al., (2015), Schumacher et al., (2016).

\begin{tabular}{ll}
\hline Taxon & Score \\
\hline Asthenocormus & 1001000000 \\
Australopachycormus & 0010000000 \\
Bonnerichthys & 0101000001 \\
Euthynotus & 1000000000 \\
Hypocormus & 1000000000 \\
Leedsichthys & 1000000100 \\
Martillichthys & 1000000000 \\
Ohmdenia & 1000000000 \\
Orthocormus & 1000000000 \\
Pachycormus & 1000000000 \\
Protosphyraena & 1100010010 \\
Rhinconichthys & 1101000000 \\
Saurostomus & 1000000000 \\
\hline
\end{tabular}




\section{LITERATURE CITED}

Arratia, G., and H.-P Schultze. 2013. Outstanding features of a new Late Jurassic pachycormiform fish from the Kimmeridgian of Brunn, Germany and comments on current understanding of pachycormiforms; pp. 87-120 in G. Arratia, H.-P Schultze, and M. V. H. Wilson (eds.), Mesozoic Fishes 5 - Global Diversity and Evolution. Verlag Dr. Friedrich Pfeil, München.

Bazzi, M., E. Einarsson, and B. P. Kear. 2015. Late Cretaceous (Campanian) actinopterygian fishes from the Kristianstad Basin of southern Sweden; DOI:10.1144/SP434.5 in B. P. Kear, J. Lundgren, J. H. Hurum, J. Milàn, and V. Vajda (eds.), Mesozoic Biotas of Scandinavia and its Arctic Territories. Geological Society, London, Special Publications 434.

Friedman, M., K. Shimada, L. D. Martin, M. J. Everhart, J. Liston, A. Maltese, and M. Triebold. 2010. 100-million year dynasty of giant planktivorous bony fishes in the Mesozoic seas. Science 327:990-993.

Friedman, M. 2012. Parallel evolutionary trajectories underlie the origin of giant suspensionfeeding whales and bony fishes. Proceeding of the Royal Society B 279:944-951.

Friedman, M., K. Shimada, M. J. Everhart, K. J. Irwin, B. S. Grandstaff, and J. D. Stewart. 2013. Geographic and stratigraphic distribution of the Late Cretaceous suspension-feeding bony fish Bonnerichthys gladius (Teleostei, Pachycormiformes). Journal of Vertebrate Paleontology 33:35-47.

Kear, B. 2007. First record of a pachycormid fish (Actinopterygii: Pachycormiformes) from the lower Cretaceous of Australia. Journal of Vertebrate Paleontology 27:1033-1038.

Kear, B. P., T. H. Rich, M. A. Ali, Y. A. Al-Mufarrih, A. H. Matiri, A. M. Al-Masary, and Y. Attia. 2009. An Upper Cretaceous (Campanian-Maastrichtian) actinopterygian fish assemblage from the marginal marine Adaffa Formation of Saudi Arabia. Cretaceous Research 30: 1164-1168.

Lambers P. 1992. On the Ichthyofauna of the Solnhofen litographic limestone (Upper Jurassic, Germany). Unpublished Ph.D. dissertation, University of Groningen, Groningen, The Netherlands, $336 \mathrm{pp}$.

Liston, J. 2010. The occurrence of the Middle Jurassic pachycormid fish Leedsichthys. Oryctos $1-36$.

McNamara, K. H., D. Friend, and J. Long. 1993. A Guide to fossils of the Gingin Chalk. Western Australian Museum, Perth, 16 pp.

Mainwaring, A. J. 1978. Anatomical and systematic revision of the Pachycormidae, a family of Mesozoic fossil fishes. Unpublished Ph.D. dissertation, Westfield College, London, 162 pp.

Schumacher, B. A., K. Shimada, J. Liston, \& A. Maltese. 2016. Highly specialized suspension-feeding bony fish Rhinconichthys (Actinopterygii: Pachycormiformes) from the mid-Cretaceous of the United States, England, and Japan. Cretaceous Research 61:7185.

Stewart, J. D. 1988. The stratigraphic distribution of Late Cretaceous Protosphyraena in Kansas and Alabama; pp. 80-94 in M. E. Nelson (ed.), Geology, Paleontology and Biostratigraphy of Western Kansas: Articles in Honor of Myrl V. Walker (Fort Hays Studies, Third Series (Science) Number 10). Fort Hays State University, Hays, Kansas. 